\title{
One-year outcomes of the Surgical Treatment of Aortic Stenosis With a Next Generation Surgical Aortic Valve (TRITON) trial: A prospective multicenter study of rapid-deployment aortic valve replacement with the EDWARDS INTUITY Valve System
}

\author{
Alfred A. Kocher, MD, PhD, ${ }^{a}$ Günther Laufer, MD, PhD, ${ }^{a}$ Axel Haverich, MD, PhD, ${ }^{b}$ \\ Malakh Shrestha, MD, PhD, MBBS, ${ }^{\mathrm{b}}$ Thomas Walther, MD, PhD, ${ }^{\mathrm{c}}$ Martin Misfeld, MD, PhD, ${ }^{\mathrm{d}}$ \\ Joerg Kempfert, MD, ${ }^{\mathrm{c}}$ Linda Gillam, MD, MPH, ${ }^{\mathrm{e}}$ Christoph Schmitz, MD, ${ }^{\mathrm{f}}$ Thorsten C. Wahlers, MD, \\ $\mathrm{PhD},{ }^{\mathrm{g}}$ Jens Wippermann, MD, ${ }^{\mathrm{g}}$ Friedrich W. Mohr, MD, PhD,${ }^{\mathrm{d}}$ Matthias Roth, MD, ${ }^{\mathrm{c}}$ \\ Adalbert Skwara, MD, ${ }^{\mathrm{c}}$ Parwis Rahmanian, MD, ${ }^{\mathrm{g}}$ Dominik Wiedemann, $\mathrm{MD},{ }^{\mathrm{a}}$ and \\ Michael A. Borger, MD, $\mathrm{PhD}^{\mathrm{d}}$
}

\begin{abstract}
Objectives: A new class of rapid-deployment aortic valves has emerged with the potential to simplify minimally invasive aortic surgery and reduce crossclamp and cardiopulmonary bypass times. We report the 1-year clinical outcomes of aortic valve replacement with the EDWARDS INTUITY Valve System (Edwards Lifesciences LLC, Irvine, Calif) in the Surgical Treatment of Aortic Stenosis With a Next Generation Surgical Aortic Valve (TRITON) trial.
\end{abstract}

\begin{abstract}
Methods: Seventeen surgeons from 6 European centers treated 152 consecutive patients with aortic stenosis requiring valve replacement in a prospective, single-arm trial. A stented trileaflet bovine pericardial bioprosthesis with a balloon-expandable, cloth-covered stent frame at the inflow aspect was implanted in 146 patients (mean age, $75.5 \pm 6.7$ years; $52.7 \%$ were female). Five valve sizes were evaluated $(19-27 \mathrm{~mm}) ; 58.9 \%$ of cases had isolated aortic valve replacement, and $41.1 \%$ of cases involved concomitant procedures. Minimally invasive surgical approaches occurred in $48.8 \%$ of the isolated aortic valve replacements. Patients were followed at discharge, 3 months, and 1 year postoperatively.
\end{abstract}

Results: Implantation success was $96.1 \%$ (146/152), early valve-related mortality was $1.4 \%(2 / 146)$, and cumulative survival was $92.5 \%$ at a mean follow-up of $9.8 \pm 5.1$ months. Crossclamp time for isolated aortic valve replacement was $41.1 \pm 10.6$ minutes. Independent core laboratory-adjudicated mean effective orifice area and aortic valve pressure gradient were $1.7 \pm 0.2 \mathrm{~cm}^{2}$ and $8.8 \pm 3.0 \mathrm{~mm} \mathrm{Hg}$ at 3 months, and $1.7 \pm 0.2$ $\mathrm{cm}^{2}$ and $8.4 \pm 3.4 \mathrm{~mm} \mathrm{Hg}$ at 1 year, respectively.

Conclusions: Implantation of the EDWARDS INTUITY Valve System is feasible, safe, and efficacious for aortic valve replacement. Aortic crossclamp and cardiopulmonary bypass times were reduced compared with those for conventional aortic valve replacement. Early hemodynamic performance was excellent and remained so up to 1 year. (J Thorac Cardiovasc Surg 2013;145:110-6)

Surgical aortic valve replacement (AVR) with cardiopulmonary bypass (CPB) has been the treatment of choice for symptomatic aortic stenosis for more than 50 years. Major

\footnotetext{
From the Department of Cardiac Surgery, ${ }^{\text {a }}$ Medical University of Vienna, Vienna,

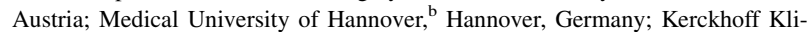
nik, ${ }^{\mathrm{c}}$ Bad Nauheim, Germany; University of Leipzig, ${ }^{\mathrm{d}}$ Leipzig, Germany; Columbia University Medical Center, ${ }^{\mathrm{e}}$ New York, NY; University of Munich, ${ }^{\mathrm{f}}$ Munich, Germany; and Medical University of Cologne, ${ }^{\mathrm{g}}$ Cologne, Germany.

Disclosures: Authors have nothing to disclose with regard to commercial support.

Read at the 92nd Annual Meeting of The American Association for Thoracic Surgery, San Francisco, California, April 28-May 2, 2012.

Received for publication April 14, 2012; revisions received June 22, 2012; accepted for publication July 26, 2012; available ahead of print Oct 10, 2012.

Address for reprints: Alfred A. Kocher, MD, PhD, Department of Cardiac Surgery, General Hospital Vienna, Medical University Vienna, Waehringerguertel 18-20, 1090 Vienna, Austria (E-mail: Alfred.Kocher@meduniwien.ac.at). $0022-5223 / \$ 36.00$

Copyright (c) 2013 by The American Association for Thoracic Surgery http://dx.doi.org/10.1016/j.jtcvs.2012.07.108
}

advances in valve technology have led to considerable improvements in both valve design and surgical implant technique of aortic bioprostheses. Enhanced structural design modifications to achieve increased effective orifice area (EOA) and reduced strut height have led to superior hemodynamics. ${ }^{1-4}$ Moreover, advancements in aortic valve design have facilitated minimally invasive surgical approaches while achieving reductions in procedural times. ${ }^{5}$ Although transcatheter AVR has expanded the treatment options for aortic stenosis, this therapy is currently reserved for patients who are ineligible for traditional AVR surgical approaches because of advanced age, poor left ventricular systolic function, severe comorbidities, and high-risk anatomic characteristics. ${ }^{6,7}$

A novel rapid-deployment AVR (RDAVR) technique has recently been introduced for conventional AVR in patients with aortic stenosis. The EDWARDS INTUITY Valve 


\section{Abbreviations and Acronyms \\ AVR = aortic valve replacement \\ $\mathrm{CABG}=$ coronary artery bypass grafting \\ $\mathrm{CPB}=$ cardiopulmonary bypass \\ EOA = effective orifice area \\ NYHA $=$ New York Heart Association \\ RDAVR $=$ rapid-deployment aortic valve replacement \\ TRITON $=$ Surgical Treatment of Aortic Stenosis With a Next Generation Surgical Aortic Valve trial}

System (Edwards Lifesciences LLC, Irvine, Calif) is designed for RDAVR and is built on the proven long-term safety and efficacy of the Carpentier-Edwards Perimount (Edwards Lifesciences LLC) family of valves while leveraging recent design innovation from Edwards' transcatheter heart valves. The EDWARDS INTUITY Valve System consists of a bioprosthesis, delivery system, and balloon catheter, which is used to deploy the valve after placement within the aortic annulus. Clinical benefits of RDAVR may include reduced operative times, decreased morbidity, faster recovery, and less-invasive surgical options for patients undergoing valve replacement. ${ }^{8,9}$

The current study presents the 1-year clinical outcomes and device efficacy of the first 146 subjects receiving an EDWARDS INTUITY Valve in a prospective, multicenter study (Surgical Treatment of Aortic Stenosis With a Next Generation Surgical Aortic Valve [TRITON], ClinicalTrials.gov number, NCT01445171). The purpose of the TRITON trial was to evaluate the safety, feasibility, and efficacy of the EDWARDS INTUITY Valve System.

\section{METHODS AND MATERIALS \\ Study Population}

Seventeen surgeons from 6 European centers treated 152 consecutive patients with aortic stenosis requiring elective valve replacement in a nonrandomized, single-arm trial. The trial was designed by members of the executive committee, which included 1 academic principal investigator and 6 cardiac surgeons, in collaboration with the sponsor (Edwards Lifesciences LLC). The sponsor funded the study and managed collection and monitoring of the data. The ethics committee of each center approved the protocol, and all patients gave written informed consent. Trial inclusion and exclusion criteria are listed in Appendix 1. Consenting patients received the EDWARDS INTUITY Valve System (Model 8300A), a stented trileaflet bovine pericardial bioprosthesis with a balloon-expandable, cloth-covered stent frame at the inflow aspect (Figure 1).

\section{Rapid-Deployment Aortic Valve Replacement}

After standard aortotomy, the native aortic valve leaflets were excised and calcium debridement of the annulus was performed. Three equidistant guiding sutures were placed through the nadir of the aortic annulus and then placed in corresponding positions through the sewing ring of the study valve. By using the guiding sutures, the valve and attached delivery system were lowered onto the annulus and secured into position under direct vision. The balloon catheter was then inflated to deploy the stent frame in a controlled fashion. Inflation pressures ranged from 3 to 5 atm depending on the size of the prosthesis. On deployment, the prosthesis was fixed in a supra-annular position while the stent skirt frame was seated below the annulus in a flared configuration within the left ventricular outflow tract. Once the skirt frame was deployed, the delivery system and valve holder were removed as a single unit, the 3 guiding sutures were tied, and the aortotomy was closed using standard surgical techniques. Deployment time was defined as the time between the first suture placement on the bioprosthesis and the end of balloon inflation. Valve implant time was defined as the time between the first suture placement on the bioprosthesis and the removal of the delivery system. Postoperative anticoagulant therapy was recommended in accordance with the American College of Cardiology/ American Heart Association 2006 Guidelines for Management of Patients with Valvular Heart Disease. ${ }^{10}$

\section{End Points}

Safety end points included valve-related mortality, thromboembolic events, study valve thrombosis, major bleeding events, paravalvular leakage, and prosthetic valve endocarditis. Definitions are included in Appendix 2. The study protocol was guided by ISO 5840:2005 and ISO 14155:2011. Performance end points included device technical success, procedural success, deployment time, implant time, changes in New York Heart Association (NYHA) class, and echocardiographic hemodynamic performance.

\section{Follow-up}

Clinical and echocardiographic follow-up data were collected at discharge, 3 months, and 1 year postoperatively. The following procedures were performed at 3 months and 1 year: physical examination, assessment of complications, coagulation profile, transthoracic echocardiography, and blood studies. Functional assessment was performed at baseline, at 3 months, and at 1 year. Hemodynamic data were reviewed by an independent echocardiographic core laboratory (Columbia University Medical Center, New York, NY), and complications were adjudicated by an independent Clinical Events Committee. Aggregate data were reviewed by an independent Data Safety Monitoring Board.

\section{Statistics}

Continuous variables are summarized as mean and standard deviation. Categoric variables are summarized as the number and percentage of subjects in each category. Complications were summarized for the early $(\leq 30$ days postoperatively) and late ( $>30$ days postoperatively) periods. Early rates are calculated as the number of events divided by the number of subjects receiving implants (as-treated analysis). Linearized rates for complications are calculated for the late period as the number of late events divided by the number of late patient-years.

\section{RESULTS}

\section{Patient Demographics}

The EDWARDS INTUITY Valve System was implanted in 146 of 152 patients. Nonstudy valves were implanted in 6 patients (lack of valve size availability in 4 and aberrant annular morphology in 2). The mean age of the 146 patients was $75.5 \pm 6.7$ years (range, 51-89 years). Patients had a European System for Cardiac Operative Risk Evaluation of $7.9 \pm 6.5$, and $52.7 \%$ were female (Table 1 ).

\section{Intraoperative Findings}

Overall technical success and procedural success were achieved in $96.1 \%(146 / 152)$ and $97.3 \%(142 / 146)$ of the 


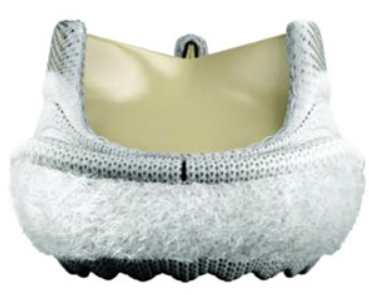

Model 8300A

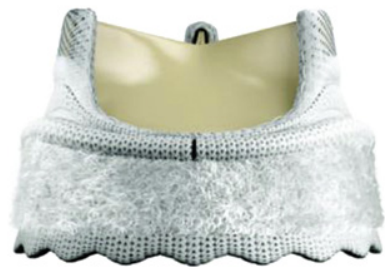

Model 8300A Deployed

FIGURE 1. Rapid-deployment aortic valve: EDWARDS INTUITY Valve System, Model 8300A (Edwards Lifesciences LLC, Irvine, Calif).

cases, respectively. The device was implanted through a full sternotomy $(69.9 \%)$ or a minimally invasive approach $(30.1 \%)$, which included an upper hemisternotomy in $97.7 \%$ of the minimally invasive surgery cases (Table 2). Isolated AVR was performed in $58.9 \%$ of the cases, and AVR with concomitant coronary artery bypass grafting (CABG) $(\times 1, \times 2, \times 3)$ was performed in $24.7 \%$ of the cases. In $16.4 \%$ of the cases, AVR was performed concomitantly with another procedure, such as carotid thromboendarterectomy $(\mathrm{n}=2)$, excision of mediastinal tumor $(\mathrm{n}=1)$, occlusion of atrioseptal defect $(\mathrm{n}=1)$, Maze procedure $(\mathrm{n}=12)$, septal myectomy $(\mathrm{n}=2)$, and occlusion of foramen ovale $(\mathrm{n}=2)$.

\section{Complications}

At 3 months and 1 year, 133 and 96 patients had completed follow-up, respectively, with a mean follow-up of $9.8 \pm 5.1$ months $(\mathrm{n}=146)$. All-cause and valve-related early mortality were $2.1 \%(3 / 146)$ and $1.4 \%(2 / 146)$, respectively. The specific causes of early mortality were reported as sepsis (non-valve related), heart failure, and cardiopulmonary failure. All-cause and valve-related late mortality were $7.5 \%$ and $1.9 \%$, respectively (Table 3 ). The specific causes of late deaths were reported as respiratory failure $(\mathrm{n}=2)$, infection $(\mathrm{n}=1)$, rupture of abdominal aortic aneurysm $(\mathrm{n}=1)$, advanced metastatic cancer $(\mathrm{n}=1)$, myocardial infarction $(\mathrm{n}=1)$, thromboembolic event $(\mathrm{n}=1)$ (valve related), and multisystem organ failure $(\mathrm{n}=1)$ (valve related). Four early $(2.7 \%)(4 / 146)$ and 2 late $(1.9 \%)$ thromboembolic events were reported. One early

TABLE 1. Baseline characteristics of patients $(n=146)$

\begin{tabular}{lc}
\hline \multicolumn{1}{c}{ Parameter } & Mean \pm SD, or $\mathbf{n}(\%)$ \\
\hline Age $(\mathrm{y})$ & $75.5 \pm 6.7$ \\
Female & $77(52.7 \%)$ \\
Risk level, logistic euroSCORE I $(\%)^{*}$ & $7.9 \pm 6.5$ \\
NYHA class III or IV & $68(46.6 \%)$ \\
\hline
\end{tabular}

SD, Standard deviation; euroSCORE, European System for Cardiac Operative Risk Evaluation; NYHA, New York Heart Association. *Logistic euroSCORE I data were not available for 4 patients.

TABLE 2. Intraoperative data

\begin{tabular}{lc}
\hline \multicolumn{1}{c}{ Parameter } & n (\%) or mean \pm SD \\
\hline Valve size $(\mathrm{mm})(\mathrm{n}=146)$ & $1(0.7 \%)$ \\
19 & $50(34.2 \%)$ \\
21 & $52(35.6 \%)$ \\
23 & $35(24.0 \%)$ \\
25 & $8(5.5 \%)$ \\
27 & \\
Procedures $(\mathrm{n}=146)$ & $86(58.9 \%)$ \\
AVR only & $36(24.7 \%)$ \\
AVR + CABG & $24(16.4 \%)$ \\
AVR + other & \\
Surgical approach $(\mathrm{n}=146)$ & $102(69.9 \%)$ \\
Full sternotomy & $44(30.1 \%)$ \\
Minimally invasive approach & $43(29.5 \%)$ \\
Upper hemisternotomy & $1(0.7 \%)$ \\
Right anterior minithoracotomy & $9.7 \pm 4.3$ \\
Deployment time $($ min $)(\mathrm{n}=133)^{*}$ & $11.0 \pm 6.6$ \\
Valve implant time $(\min )(\mathrm{n}=145) \dagger$ & $46.6 \pm 16.4$ \\
Crossclamp time $($ min $)(\mathrm{n}=134) \ddagger$ & $41.1 \pm 10.6$ \\
AVR only $(\mathrm{n}=80)$ & $60.0 \pm 19.0$ \\
AVR + CABG $(\mathrm{n}=32)$ & $47.0 \pm 19.2$ \\
AVR + other $(\mathrm{n}=22)$ & $75.1 \pm 26.4$ \\
CPB time $($ min $)(\mathrm{n}=134) \ddagger$ & $66.3 \pm 18.7$ \\
AVR only $(\mathrm{n}=80)$ & $95.6 \pm 30.4$ \\
AVR + CABG $(\mathrm{n}=32)$ & $77.2 \pm 28.1$ \\
AVR + other $(\mathrm{n}=22)$ & \\
\hline
\end{tabular}

$S D$, Standard deviation; $A V R$, aortic valve replacement; $C A B G$, coronary artery bypass grafting; $C P B$, cardiopulmonary bypass. Deployment and valve implant times are summarized for the successful implant. *In 13 cases, balloon inflation time was not reported. $\dagger$ In 1 case, delivery system removal time was not reported. $\ddagger$ Technical success in the first attempt.

$(0.7 \%)(1 / 146)$ procedure-related reoperation for major bleeding was reported. No cases of coronary ostial obstruction or prosthesis interference with aortotomy closure were observed. Two early ( 1 mild and $1 \mathrm{mild} /$ moderate) cases of paravalvular leak $(1.4 \%)(2 / 146)$ that remained unchanged over 1 year were reported. In addition, 1 late moderate/severe paravalvular leak $(0.9 \%)$ was reported at

TABLE 3. Early and late complications

\begin{tabular}{lcc}
\hline \multicolumn{1}{c}{ Parameter } & $\begin{array}{c}\text { Early }(\leq \mathbf{3 0} \text { d) } \\
(\mathbf{n}=\mathbf{1 4 6}) \\
\mathbf{N}(\%)\end{array}$ & $\begin{array}{c}\text { Late }(>\mathbf{3 0} \mathbf{d}) \\
\text { (late patient-y }=\mathbf{1 0 7 . 2 8}) \\
\mathbf{N}(\%)\end{array}$ \\
\hline Mortality & & \\
$\quad$ All cause & $3(2.1 \%)$ & $8(7.5 \%)$ \\
$\quad$ Valve related & $2(1.4 \%)$ & $2(1.9 \%)$ \\
Thromboembolism & $4(2.7 \%)$ & $2(1.9 \%)$ \\
Reoperation for bleeding & $1(0.7 \%)$ & $0(0.0 \%)$ \\
Paravalvular leak $(>1+)$ & $2(1.4 \%)$ & $1(0.9 \%)$ \\
Explant & $2(1.4 \%)$ & $2(1.9 \%)$ \\
Endocarditis & $0(0.0 \%)$ & $0(0.0 \%)$ \\
Hemolysis & $0(0.0 \%)$ & $0(0.0 \%)$ \\
Structural valve deterioration & $0(0.0 \%)$ & $0(0.0 \%)$ \\
\hline
\end{tabular}

$\mathrm{N}$ represents the number of events. 
postoperative day 92 , which led to a reoperation with explant of the prosthetic valve. At explant, the annulus appeared circumferentially irregular with a notch in the left coronary-right coronary commissure region that corresponded to the paravalvular leak observed at the 3-month echocardiographic assessment. Two early $(1.4 \%)(2 / 146)$ and 2 late $(1.9 \%)$ explants were reported (all were procedure and valve related). The specific causes of early explant were reported as bleeding during and after the procedure because of aortic dissection and cardiogenic shock leading to valve damage secondary to manual cardiopulmonary resuscitation. The specific causes of late explants were reported as worsening paravalvular leak from moderate to severe and pseudoaneurysm of the left ventricular outflow tract observed at the 3-month echocardiographic assessment. At the time of explant, the patient was asymptomatic with good valve function and absence of paravalvular leak or insufficiency. No endocarditis, hemolysis, or structural valve deterioration was observed.

Early permanent pacemaker implant was required in 10 patients and adjudicated as study valve related in 7 patients $(5.0 \%)(7 / 141)$ (Table 4$)$. Of note, all early valve-related permanent pacemaker implants had preexisting conduction issues. Preexisting conduction disturbances included atrial fibrillation $(\mathrm{n}=4)$, atrioventricular block (I and II) $(\mathrm{n}=5)$, and right or left bundle branch block $(\mathrm{n}=5)$. Late permanent pacemaker implant occurred in 3 cases, of which $1(0.8 \%)$ (1/127) was valve related. The 2 non-valve-related late cases involved worsening of a preexisting conduction disturbance.

\section{Device Performance}

Reported mean EOA was $1.7 \pm 0.2 \mathrm{~cm}^{2}$ at discharge $(\mathrm{n}=90)$ and remained unchanged at 3 months $(\mathrm{n}=99)$ and 1 year $(\mathrm{n}=65)$. Mean and peak systolic gradients were $9.8 \pm 3.3 \mathrm{~mm} \mathrm{Hg}$ and $18.7 \pm 6.5 \mathrm{~mm} \mathrm{Hg}$ at discharge, respectively $(\mathrm{n}=108)$. Mean and peak pressure gradients were $8.8 \pm 3.0 \mathrm{~mm} \mathrm{Hg}$ and $16.7 \pm 6.0 \mathrm{~mm} \mathrm{Hg}$ at 3 months $(\mathrm{n}=106)$, respectively, and decreased to $8.4 \pm 3.4 \mathrm{~mm} \mathrm{Hg}$ and $15.8 \pm 5.7 \mathrm{~mm} \mathrm{Hg}$ at 1 year $(\mathrm{n}=68)$, respectively.

The majority of patients showed improvement $(75.0 \%)$ $(99 / 132)$ or remained in the same NYHA class $(22.7 \%)$ at
3 months postoperatively, with $93.2 \%$ (123/132) of patients in NYHA class I or II. At 1 year, patients showed continued improvement $(75.0 \%)(72 / 96)$ or remained the same $(21.9 \%)$, with $96.9 \%$ (93/96) of patients having class I or II symptoms.

\section{DISCUSSION}

The 1-year outcomes from a 152-patient cohort undergoing AVR with the EDWARDS INTUITY Valve System demonstrate that the valve is safe, feasible, and efficacious. RDAVR with the EDWARDS INTUITY Valve System yields a high technical success rate of $96.1 \%$, with flexibility in procedural approach ranging from full sternotomy to upper hemisternotomy to right anterior mini-thoracotomy. Minimally invasive techniques offer the benefit of pain and scar reduction, along with shorter ventilation times, shorter intensive care unit and hospital lengths of stay, less blood loss, and faster return to routine life activities. ${ }^{8,9,11}$ In this study, RDAVR with the EDWARDS INTUITY Valve System facilitated minimally invasive surgical approaches in $48.8 \%(42 / 86)$ of the isolated AVRs. In 2009, Gummert and colleagues ${ }^{12}$ reported, from the German Database, that only $10.2 \%$ of the isolated AVRs were performed using the minimally invasive approach.

Previous studies have identified prolonged crossclamp time as a predictor of major postoperative mortality, morbidity, and length of hospital stay in both low- and high-risk patients. ${ }^{13,14}$ Salis and colleagues ${ }^{15}$ identified prolonged crossclamp and bypass times as independent predictors of morbidity and mortality after cardiac surgery in a cohort of more than 5000 patients. In 2 separate cohorts totaling more than 3200 patients, Dewey and colleagues ${ }^{16}$ reported crossclamp times in the range of 86 to 88 minutes with an early mortality rate of $5.3 \%$ in all AVRs, whereas Chan and colleagues ${ }^{17}$ reported crossclamp times for all AVRs ranging from 82 to 84 minutes with an early mortality rate of $5.0 \%$. As previously discussed, the crossclamp time in our experience was $47 \pm 16$ minutes with an early mortality rate of $1.4 \%$. In this 12 -month experience, rates for early thromboembolic events, paravalvular leak, and permanent pacemaker implant of $2.7 \%, 1.4 \%$, and $5.0 \%$,

TABLE 4. Postoperative permanent pacemaker implants

\begin{tabular}{llccc}
\hline Time period & Patient description & $\begin{array}{c}\text { No baseline conduction } \\
\text { disturbance n (\%) }\end{array}$ & $\begin{array}{c}\text { Previous conduction } \\
\text { disturbance n (\%) }\end{array}$ & All subjects n (\%) \\
\hline$\leq 30 \mathrm{~d}$ & No. of patients at risk & 102 & 39 & 141 \\
& Valve related & $0(0.0 \%)$ & $7(17.9 \%)$ & $7(5.0 \%)$ \\
& Non-valve related & $1(1.0 \%)$ & $2(5.1 \%)$ & $3(2.1 \%)$ \\
$10 \mathrm{~d}$ & No. of patients at risk & 98 & 29 & 127 \\
& Valve related & $1(1.0 \%)$ & $0(0.0 \%)$ & $1(0.8 \%)$ \\
& Non-valve related & $0(0.0 \%)$ & $2(6.9 \%)$ & $2(1.6 \%)$ \\
\hline
\end{tabular}

Patients at risk have reached the start of the interval without a permanent pacemaker implant. Percentages are based on the number of patients with a postoperative pacemaker implant by the end of the interval divided by the number at risk at the start of the interval. Patients with "no baseline conduction disturbances" are those with "sinus" at the baseline electrocardiogram with no other conditions checked. Patients with a baseline pacemaker or pacemaker implanted intraoperatively are not included in this analysis. 
respectively, compare favorably with reported range rates for conventional AVR of $2.7 \%$ to $3.9 \%, 5.6 \%$ to $6 \%$, and $3 \%$ to $11.8 \%$, respectively. ${ }^{16,18-21}$

Reproducible crossclamp and CPB times were demonstrated in isolated RDAVR and complex RDAVR. We report crossclamp times of $41 \pm 11$ minutes and $60 \pm 19$ minutes for RDAVR and RDAVR + CABG, respectively. CPB times were $66 \pm 19$ minutes and $96 \pm 30$ minutes for RDAVR and RDAVR + CABG, respectively. The EPICARD Database reported crossclamp times of 57 and 87 minutes for isolated AVR and AVR +CABG, respectively. ${ }^{22}$ CPB times were 76 and 113 minutes for isolated AVR and AVR + CABG, respectively. In a 1000-patient cohort, McClure and colleagues $^{23}$ report crossclamp times of $75 \pm 1$ minutes and $98 \pm 2$ minutes for AVR and AVR + CABG, respectively. CPB times for AVR and AVR $+\mathrm{CABG}$ were reported as $106 \pm 2$ minutes and $136 \pm 2$ minutes, respectively. Thus, when compared with conventional AVR procedural times from the study by McClure and colleagues, mean reductions in crossclamp time for isolated RDAVR and RDAVR + CABG were $45 \%$ and $39 \%$, respectively, whereas mean reductions in CPB times for RDAVR and RDAVR + CABG were $38 \%$ and $29 \%$, respectively.

The design concept of the EDWARDS INTUITY Valve System is based on the Carpentier-Edwards Perimount valve. To date, more than 500,000 Perimount aortic valves have been implanted in patients. ${ }^{24,25}$ The hallmarks of the Perimount valve include a low profile, supra-annular design, and unique anticalcification technology. The hemodynamic performance of the Perimount valve has been reported. ${ }^{2,3}$ The hemodynamic performance of the EDWARDS INTUITY Valve System favorably correlates with the hemodynamic performance reported in the literature for Perimount valves. Dalmau and colleagues ${ }^{3}$ reported a mean EOA and pressure gradient of $1.9 \pm 0.4 \mathrm{~cm}^{2}$ and $10.3 \pm 3.4 \mathrm{~mm} \mathrm{Hg}$ at 1 year, respectively, whereas Cohen and colleagues ${ }^{2}$ reported a mean EOA and pressure gradient of $1.9 \pm 0.6 \mathrm{~cm}^{2}$ and $7.1 \pm 3.7 \mathrm{~mm} \mathrm{Hg}$ at 1 year, respectively. Furthermore, Cohen and colleagues demonstrated favorable long-term hemodynamic performance of the CarpentierEdwards Perimount valves at 9 years, with a mean EOA and mean pressure gradient of $1.5 \pm 0.6 \mathrm{~cm}^{2}$ and $10.9 \pm$ $3.7 \mathrm{~mm} \mathrm{Hg}$, respectively. ${ }^{2}$

\section{Study Limitations}

This was a nonrandomized, single-arm study without a concurrent control group. Roll-in cases were included in these data, and a learning curve impact is not taken into account. Other important limitations may include the possibility of selection and performance biases due a nonblinded study design, as well as attrition biases resulting from missing data points. In addition, these reported data extend up to only 1 year, and long-term performance and complications may differ over an extended period of time.

\section{CONCLUSIONS}

The EDWARDS INTUITY Valve System is safe, feasible, and efficacious. RDAVR with the EDWARDS INTUITY Valve System facilitates minimally invasive surgical approaches while offering reproducible reductions in crossclamp and bypass times, which may yield potential benefits of shortened length of hospital stay, along with improvements in morbidity and mortality outcomes. Excellent hemodynamics and low complication rates are reported. Given the similarity in basic valve design, the long-term durability and hemodynamic performance of the EDWARDS INTUITY Valve System should be consistent with that of the Perimount family of valves. Further evaluation, including long-term follow-up and future studies with a prospective, randomized, controlled design, is recommended for assessment of conventional AVR and RDAVR.

\section{References}

1. Borger MA, Nette AF, Maganti M, Feindel CM. Carpentier-Edwards Perimount Magna valve versus Medtronic Hancock II. Ann Thorac Surg. 2007;83:2054-9.

2. Cohen G, Christakis GT, Joyner CD, et al. Are stentless valves hemodynamically superior to stented valves? J Thorac Cardiovasc Surg. 2010;139:848-59.

3. Dalmau MJ, Gonzalez-Santos JM, Blazquez JA, et al. Hemodynamic performance of the Medtronic Mosaic and Perimount Magna aortic bioprostheses. Eur J Cardiothorac Surg. 2011;39:844-52.

4. Brown JM, O'Brien SM, Wu C, Sikora JA, Griffith BP, Gammie JS. Isolated aortic valve replacement in North America comprising 108,687 patients in 10 years. J Thorac Cardiovasc Surg. 2009;137:82-90.

5. Zannis K, Folliguet T, Laborde F. New sutureless aortic valve prosthesis: another tool in less invasive aortic valve replacement. Curr Opin Cardiol. 2012;27:125-9.

6. Smith CR, Leon MB, Mack MJ, et al. Transcatheter versus surgical aortic-valve replacement in high-risk patients. N Engl J Med. 2011;364:2187-98.

7. Miller DC, Blackstone EH, Mack MJ, et al. Transcatheter (TAVR) versus surgical (AVR) aortic valve replacement. J Thorac Cardiovasc Surg. 2012;2012:832-43.

8. Doll N, Borger MA, Hain J, et al. Minimal access aortic valve replacement: effects on morbidity and resource utilization. Ann Thorac Surg. 2002;74:S1318-22.

9. Tabata M, Umakanthan R, Cohn LH, et al. Early and late outcomes of 1000 minimally invasive aortic valve operations. Eur J Cardiothorac Surg. 2008;33:537-41.

10. Bonow RO, Carabello BA, Kanu C, et al. ACC/AHA 2006 guidelines for the management of patients with valvular heart disease. Circulation. 2006;114: e84-231.

11. Dogan S, Dzemali O, Wimmer-Greinecker G, et al. Minimally invasive versus conventional aortic valve replacement. J Heart Valv Dis. 2003;12:76-80.

12. Gummert J, Funkat A, Beckman A, et al. Cardiac surgery in Germany during 2009. A report on behalf of the German Society for Thoracic and Cardiovascular Surgery. Thorac Cardiovasc Surg. 2010;58:379-86.

13. Al-Sarraf N, Thalib L, Hughes A, et al. Cross-clamp time is an independent predictor of mortality and morbidity in low- and high-risk cardiac patients. Int J Surg. 2011;9:104-9.

14. Bagur R, Manazzoni JM, Dumont E, et al. Permanent pacemaker implantation following isolated aortic valve replacement in a large cohort of elderly patients with severe aortic stenosis. Heart. 2011;97:1687-94.

15. Salis S, Mazzanti VV, Merli G, et al. Cardiopulmonary bypass duration is an independent predictor of morbidity and mortality after cardiac surgery. $J$ Cardiothorac Vasc Anesth. 2008;22:814-22.

16. Dewey TM, Herbert MA, Ryan WH, et al. Influence of surgeon volume on outcomes with aortic valve replacement. Ann Thorac Surg. 2012;93:1107-13.

17. Chan V, Kulik A, Tran A, et al. Long-term clinical and hemodynamic performance of the Hancock II versus the Perimount aortic bioprosthesis. Circulation. 2010;122:S10-6.

18. Jamieson WRE, Moffatt-Bruce SD, Skarsgard P, et al. Early antithrombotic therapy for aortic valve bioprosthesis. Ann Thorac Surg. 2007;83:549-57.

19. Davila-Roman VG, Waggoner AD, Kennard ED, et al. Prevalence and Severity of Paravalvular Regurgitation in the Artificial Valve Endocarditis Reduction Trial (AVERT) Echocardiography Study. J Am Coll Cardiol. 2004;44:1467-72. 
20. Ionescu A, Fraser AG, Butchart EG. Prevalence and clinical significance of incidental paraprosthetic valvar regurgitation. Heart. 2003;83:1316-21.

21. Matthews IG, Fazal IA, Bates MG, Turley AJ. In patients undergoing aortic valve replacement, what factors predict the requirement for permanent pacemaker implantation? Interact Cardiovasc Thorac Surg. 2011;12:475-9.

22. EPICARD Database, Thoracic and Cardiovascular Surgery. 6th ed. Paris, France: The French Society of Thoracic and Cardiovascular Surgery; 2009.

23. McClure RS, Narayanasamy N, Wiegerinck E, et al. Late outcomes for aortic valve replacement with the Carpentier-Edwards pericardial bioprosthesis: up to 17-year follow-up in 1,000 patients. Ann Thorac Surg. 2010;89:1410-6.

24. Frater RWM, Salomon NW, Rainer WG, Cosgrove DM III, Wickham E. The Carpentier-Edwards pericardial aortic valve: intermediate results. Ann Thorac Surg. 1992;53:764-71

25. Le Tourneau T, Vincentelli A, Fayad G, et al. Ten-year echocardiographic and clinical follow-up of aortic Carpentier-Edwards pericardial and supraannular prosthesis. Ann Thorac Surg. 2002;74:2010-5

\section{APPENDIX 1. INCLUSION AND EXCLUSION CRITERIA}

TRITON trial inclusion criteria were as follows: patient aged 18 years or more; patient who has aortic stenosis or stenosis insufficiency of an aortic valve requiring a planned replacement as indicated in the preoperative evaluation; patient who is scheduled to undergo planned AVR with or without concomitant coronary bypass surgery; patient who has signed and dated the study informed consent form before study procedures; and patient who is geographically stable and agrees to attend follow-up assessments at the hospital of surgical services for a maximum of 5 years.

TRITON trial exclusion criteria were as follows: patient with pure aortic insufficiency; patient who requires emergency surgery; patient with an aneurysm of the aortic root or ascending aorta requiring surgical intervention; patient with a left ventricular ejection fraction less than $25 \%$; patient with a congenital bicuspid aortic valve; patient who has had active endocarditis within 3 months before the scheduled AVR surgery; patient with concomitant valve (mitral, tricuspid, pulmonic) disease requiring repair with an annuloplasty ring or replacement with a prosthesis; patient who has had prior mitral prior mitral, tricuspid, or pulmonic valve surgery, which included implantation of a bioprosthetic valve, mechanical valve, or annuloplasty ring that will remain in situ; patient who has had a myocardial infarction within 1 month before the scheduled AVR surgery; patient who has a noncardiac disease limiting life expectancy to less than 12 months; patient who previously received an EDWARDS INTUITY (Edwards Lifesciences LLC, Irvine, Calif) valve implant; patient who is an intravenous drug abuser; patient who is female or lactating; patient who is currently participating in another drug or device clinical investigation; and patient with documented blood diathesis.

\section{APPENDIX 2. END POINT DEFINITIONS}

Embolism is defined as a free-flowing blood clot or lesion material that is located in the systemic or pulmonary circulation that occurs in the absence of infection after immediate perioperative period. It may be manifested as a neurologic event or noncerebral embolic event; a noncerebral event is an embolus documented operatively, clinically, or during autopsy that produces signs and symptoms due to partial or complete obstruction of a peripheral artery. The event excludes postoperative myocardial infarction unless detected by operation, clinical imaging, autopsy, or emboli due to nonthrombotic material (atherosclerosis, myxoma).

Valve thrombosis is defined as a blood clot not associated with infection, causing dysfunction of the heart valve substitute. Diagnosis is confirmed by echocardiography, angiocardiography or magnetic resonance imaging, operation, explant, or autopsy.

Bleeding event is defined as any episode of major internal or external bleeding that causes death, hospitalization, or permanent injury (eg, vision loss) or necessitates transfusion. Major bleeding unexpectedly associated with minor trauma should be reported as a bleeding event, but bleeding associated with major trauma or a major operation is considered secondary to those events and should not be reported. Bleeding events are reported for all patients regardless of whether they are taking anticoagulants or antiplatelet drugs. Although total bleeding events must be reported, bleeding events also can be reported separately for those who are taking anticoagulants or antiplatelet agents and those who are not.

Paravalvular leak is defined as a clinically or hemodynamically detectable defect between the heart valve substitute and the patient's annulus.

Endocarditis is an inflammation or infection of the endocardium, which is the inner lining of the heart muscle and, most commonly, the heart valves, typically caused by bacterial infection but can be caused by fungus. An infection for which no source is identified and may or may not be associated with classic signs of endocarditis (eg, red blood cell casts in urine, splinter hemorrhages in finger nails, roof of mouth, lesions on retina) and may or may not be associated with a vegetation inside the atrium or on a valve. Event must be confirmed by 2 consecutive positive blood cultures or imaging study, explant, or autopsy.

Technical success was defined as delivery and deployment of the study valve within 2 attempts.

Procedural success was defined as technical success and the absence of complications requiring device reoperation, permanent pacemaker implant (with baseline sinus rhythm and no conduction issues), or death.

\section{Discussion}

Dr G. Hossein Almassi (Milwaukee, Wis). You have a high incidence of primary pacemaker implantation. Is it something related to the design of the valve or the pressure at implantation of the valve? Do you have any insight into that? 
Dr Laufer. All patients who came out of the operating room and had finally been implanted with a pacemaker after the operation had a pre-existing conduction disturbance, and if you study the published data, they report a rate of $5 \%$ to $11 \%$ of permanent pacemaker insertion after isolated aortic valve replacement. However, in these cases, we also had isolated aortic and as aortic valve replacement plus CABG. So, I would say the profile of the patient was, as evident by the European System for Cardiac Operative Risk Evaluation, quite high.

Dr Harald C. Eichstaedt (Oldenburg, Germany). I am the coauthor of the following report, which discusses a different type of sutureless valve. My question is related to the incidence of thromboembolic events that you have shown. Four patients had a stroke in the early postoperative period and two later on. I would like to ask you to explain your postoperative anticoagulation regimen.

Dr Laufer. The postoperative anticoagulation regimen was low-molecular-weight heparin until the patients were switched to warfarin (Coumadin), if there were no contraindications.

Dr Christopher Young (London, United Kingdom). I would like to ask you briefly what you think the learning curve is for the insertion of this.

Dr Laufer. This is a very good question. The learning curve in this situation for 17 surgeons was there, because it was a completely new product. It is important to note that we photographed the implantation procedure in the first cases. We evaluated the inflated stent below the valve with either a dental mirror or, in some cases, with an endoscope to learn more about how the valve sits in the outflow tract. The isolated aortic cases can be done with a crossclamp time of 20 to 30 minutes.

Dr Young. Studying all those subgroups of ministernotomy, full sternotomy, AVR plus, and AVR on its own, do you have a sense for which group this had the largest impact in, who goes to intensive care unit quicker, who goes home earliest? Where is the big effect?

Dr Laufer. It is too early to determine which group is the one that profits most from this new valve, but basically all patients will benefit, because the procedure time is substantially shorter, the crossclamp time is substantially shorter. We do not have pledgets. I could not show, because the time was too short, the outflow aspect from the ventricular side. We have no pledgets below the valve. Thus, the hemodynamics are really superior. Also, compared with a standard Magna valve, it is very easy to close the aortotomy, because the valve is hooked up below the annulus and not with stitches at the annulus.

Dr Charles R. Bridges (Charlotte, $N C$ ). Your mean crossclamp time was 41 minutes. I was wondering whether during the course of the study you noticed a decline in the crossclamp time so we could have a better idea of the crossclamp time one could expect after the learning curve.

Dr Laufer. A very good question, but we did not study these statistics. 